\title{
Exploring High-resolution Magic Angle Spinning (HR-MAS) NMR Spectroscopy for Metabonomic Analysis of Apples
}

\author{
Martina Vermathen ${ }^{\star}$, Mattia Marzoratia , and Peter Vermathen ${ }^{\mathrm{b}}$
}

\begin{abstract}
Classical liquid-state high-resolution (HR) NMR spectroscopy has proved a powerful tool in the metabonomic analysis of liquid food samples like fruit juices. In this paper the application of ${ }^{1} \mathrm{H}$ high-resolution magic angle spinning (HR-MAS) NMR spectroscopy to apple tissue is presented probing its potential for metabonomic studies. The ${ }^{1} \mathrm{H}$ HR-MAS NMR spectra are discussed in terms of the chemical composition of apple tissue and compared to liquid-state NMR spectra of apple juice. Differences indicate that specific metabolic changes are induced by juice preparation. The feasibility of HR-MAS NMR-based multivariate analysis is demonstrated by a study distinguishing three different apple cultivars by principal component analysis (PCA). Preliminary results are shown from subsequent studies comparing three different cultivation methods by means of PCA and partial least squares discriminant analysis (PLS-DA) of the HR-MAS NMR data. The compounds responsible for discriminating organically grown apples are discussed. Finally, an outlook of our ongoing work is given including a longitudinal study on apples.
\end{abstract}

Keywords: Apple $\cdot$ HR-MAS NMR $\cdot$ Metabonomics $\cdot$ Multivariate analysis $\cdot$ PCA

\section{Introduction}

Conventional liquid-state high-resolution NMR spectroscopy has been widely recognized as powerful tool for the chemical analysis not just of single compounds but also of complex mixtures. Thus, the possibility of obtaining fingerprints and possibly simultaneously identifying and quantifying many different components in a mixture have made NMR spectroscopy a very attractive tool for metabonomic studies. ${ }^{[1,2]}$ Besides sensitivity, the extent of detectable compounds is limited by the spectral resolution which in turn depends on the applied magnetic field strength and the NMR signal line shapes. Due to their

\footnotetext{
${ }^{\star}$ Correspondence: Dr. M. Vermathen ${ }^{\mathrm{a}}$ Tel.: +41316313948

E-mail: martina.vermathen@ioc.unibe.ch aUniversity of Bern

Department of Chemistry \& Biochemistry

Freiestrasse 3

$\mathrm{CH}-3012$ Bern

bUniversity of Bern \& Inselspital

Department of Clinical Research (AMSM)

MR-Center 1

$\mathrm{CH}-3010$ Bern
}

rapid isotropic motions, liquid solutions of dissolved components usually yield sharp NMR signals, which is not the case for semi-solid or solid samples. Solid-state NMR spectroscopy is a long-standing established method applied to rigid solids, which mostly makes use of cross-polarization magic angle spinning (CP-MAS) techniques for detection of low abundant nuclei like ${ }^{13} \mathrm{C} .{ }^{[3]}$ High-resolution magic angle spinning (HR-MAS) NMR spectroscopy, in turn, is applicable to semi-solid samples with restricted molecular mobility, thereby covering the gap between lowviscous liquids and rigid solids with respect to NMR spectroscopic accessibility. HR-MAS NMR evolved from solid-state NMR about 20 years ago, and has initially mainly been used in combinatorial chemistry as a method to obtain highly resolved ${ }^{1} \mathrm{H}$ NMR spectra from molecules on solid supports. ${ }^{[4]}$ In the meantime, the application of HR-MAS NMR has gained increasing interest in a broad field of semi-solid materials like emulsions, ${ }^{[5]}$ liquid crystals, ${ }^{[6]}$ gels, ${ }^{[7]}$ food samples, ${ }^{[8]}$ and biological tissues. ${ }^{[9]}$ Despite a certain degree of mobility, under static conditions, such semi-solid materials usually give rise to NMR spectra with strong line broadening and decreased resolution. The main reasons for such properties are residual anisotropic dipolar coupling between neighboring spins and bulk magnetic susceptibility (BMS) shifts. Fast spinning around an axis inclined at an angle of $54.7^{\circ}$ ('magic angle') with respect to the axis of the external magnetic field $\mathrm{B}_{0}$ (Fig. 1) can average second-rank orientation dependent effects close to or equal to zero, thereby significantly reducing the linewidth and increasing both the spectral resolution and sensitivity. ${ }^{[10]}$ Nearly all pulse sequences used for liquid samples in conventional high resolution NMR are also applicable for HR-MAS, like for example the standard 2D pulse sequences COSY, TOCSY, and HSQC as well as editing techniques on basis of molecular diffusion or relaxation times. In particular, the HR-MAS technique provides the advantage that samples can be measured directly in a non-destructive way without any preparation steps like extraction or dissolving. Thus, HR-MAS allows a comprehensive mostly uninfluenced chemical profile of semi-solid biological samples to be obtained, which can be used for metabonomic analyses.

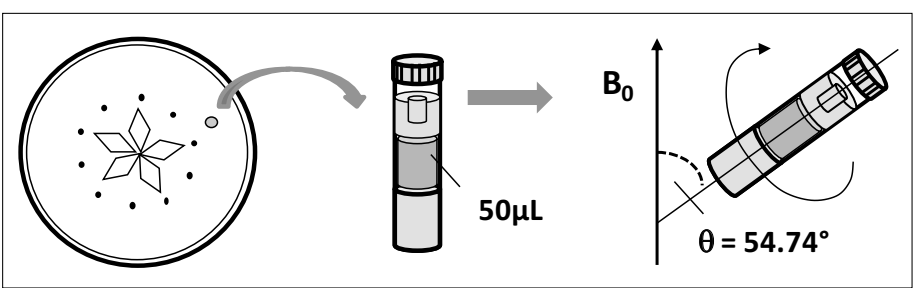

Fig. 1. Scheme of apple slice, $50 \mu \mathrm{L}$ MAS rotor with pulp sample, and rotation of the rotor at the magic angle. 
Metabonomics comprises the combined application of spectroscopy and multivariate statistical approaches to study biological multicomponent systems. ${ }^{[10,11]}$ Besides mass spectrometry, NMR spectroscopy is the most widely used method in metabonomic studies. Since the proton NMR spectra of biological samples are usually very complex, mathematical pattern recognition methods are often indispensable to detect changes or find biomarkers which might not emerge by simple visual inspection. In multivariate statistical analysis, the spectra are subdivided by a grid into regions or 'buckets' and integrated leading to a data matrix with $n$ spectra each containing $m$ buckets which in turn contain different signal intensities from metabolites. Thus, each spectrum forms a data point in a multidimensional space. The most often applied principal component analysis (PCA) is an unsupervised method projecting the data into a multidimensional space based on the variations and allows finding clusters among the data points based on similarity. Partial least squares discriminant analysis (PLS-DA) is the most often applied supervised method which uses prior knowledge, e.g. assignment of spectra to predefined groups, and is used to find the spectral properties which best discriminate the groups. ${ }^{10-12]}$

In the medical field, there has been a wide range of applications of NMR-based metabonomics on biofluids, such as urine or plasma, by liquid-state NMR ${ }^{[1]}$ and biological tissue samples by HR-MAS NMR spectroscopy ${ }^{[9]}$ to study various diseases or drug responses. In food analysis, NMRbased metabonomics is a powerful tool to probe the quality and authenticity of food samples, or to reveal adulterations. Several successful applications have been reported applying liquid-state NMR spectroscopy, such as the analysis of fruit juices, ${ }^{[13,14]}$ beer, ${ }^{[15]}$ or wine. ${ }^{[16]}$ HR-MAS NMR seems to evolve as tool for metabonomic studies of semi-solid food samples ${ }^{[8]}$ like cheese ${ }^{[17]}$ and meat. ${ }^{[18]}$ To date, there are only a few studies applied to fruits or vegetables. ${ }^{[8]}$ In this paper, our investigations to explore HR-MAS NMR for metabonomic analysis of apples are summarized. The aim of this work was to probe the validity and feasibility of this method before using it as tool to study the impact of specific growth or storage conditions on the metabolic profile of apples.

Apples belong to the most consumed fresh fruits within Switzerland and thus constitute an important economic factor in agriculture. For dessert apples it is of utmost importance that organoleptic and nutritional properties meet customer's expectations. To ensure a year-round supply of high quality apples, optimal growth and storage conditions are required. It is therefore of interest to identify and monitor chemical constituents of apples, which determine quality properties. Knowledge of metabolic changes may reveal possible causes for physiological disorders like internal browning or unwanted aroma changes during storage, both factors leading to losses in fruit sales. Currently, the most important parameters assessed to estimate internal fruit quality are sugar content, acidity and fruit firmness. ${ }^{[19]}$ In this paper, the application of HR-MAS NMR as potential complementary sensor for the detailed chemical composition of apple fruits is presented.

\section{HR-MAS NMR of Apple Tissue}

For the acquisition of an HR-MASNMR spectrum, the apple tissue can be directly punched with the MAS rotor as indicated in Fig. 1. Pulp samples were taken from the central slice of the apple cross section using MAS rotors with $50 \mu \mathrm{L}$ inserts. After addition of $\mathrm{D}_{2} \mathrm{O}$ as lock solvent, the samples were measured on a Bruker Avance II $500 \mathrm{MHz}$ system equipped with a $4 \mathrm{~mm}$ HR dual inverse ${ }^{1} \mathrm{H} /{ }^{13} \mathrm{C}$ MAS probe. In Fig. 2, a typical ${ }^{1} \mathrm{H}$ HR-MAS spectrum of an apple pulp sample is shown. The spectrum was acquired with presaturation of the water resonance (noesyprld) at a magic angle spinning rate of $5 \mathrm{kHz} .128$ transients were acquired over a spectral width of 6002.4 $\mathrm{Hz}$ with a data size of $32 \mathrm{k}$ points resulting in a total experiment time of about 12 minutes. As is evident from the spectrum shown in Fig. 2, the main constituents in apple tissue are sugar compounds. The resonances appear in the region between 3 and $5.5 \mathrm{ppm}$ including the signals of $\alpha$ - and $\beta$-glucose, sucrose, and fructose. Another prominent compound is malic acid with resonances around 2.7 and $4.4 \mathrm{ppm}$. Malic acid is the major organic acid in apples mainly accounting for the low $\mathrm{pH}$ of apple tissue or juice. ${ }^{[20]}$ As opposed to the remaining spectrum, the chemical shifts of malic acid are $\mathrm{pH}$-dependent, which needs to be taken into account when performing metabonomic studies. The use of a buffer, as it is common in liquid-state NMR of fruit juices, is not well suited in the case of apple pulp since it diffuses relatively slowly and equilibrium is reached only after a long time. The NMR signals of apple pulp span a relatively high dynamic range with the sugar resonances being roughly up to 300 -fold more intense than the resonances of the minor components of the aromatic and aliphatic regions (Figs 3 and 4). Signals in the aromatic region (5.8-10 ppm) shown in Fig. 3 mainly derive from chlorogenic acid and epicatechin, which belong to the major phenolic compounds in apple tissue besides the condensed polyphenols. ${ }^{[21]}$ Condensed polyphenols are formed by non-enzymatic or enzymatic oxidation of the monomer phenols, a process also responsible for the browning of a cut apple slice when exposed to air.[22,23] Therefore, care has to be taken in the measurement of fresh apple tissue to reduce such intervention by minimizing the time of air exposure. ${ }^{[22]}$ In our set-up, the samples, after filled into the MAS rotor, remained stable for at least 24 hours, while freezing or juice preparation quickly increased the polyphenol content. Signals in the aliphatic region (0.5-2.6 ppm) shown in Fig. 4 can be assigned to the amino acids leucine, isoleucine, valine, alanine and threonine while the broader signals primarily belong to lipids. The relatively intense doublet at 1.33 ppm overlapping with threonine has been previously tentatively assigned to paraldehyde, ${ }^{[24]}$ a cyclic trimer of acetaldehyde, which in turn is also present in the apple tissue spectrum with resonances at 2.23 and $9.65 \mathrm{ppm}$. The signals of quinic acid, which is the major organic acid in unripe apples, ${ }^{[25]}$ appear around 2 ppm overlapping with the signals of its caffeic acid ester (chlorogenic acid).

To further probe the validity of HRMAS directly applied to apple tissue, the HR-MAS spectra were compared with HR liquid-state NMR spectra of corresponding apple juices freshly prepared from the

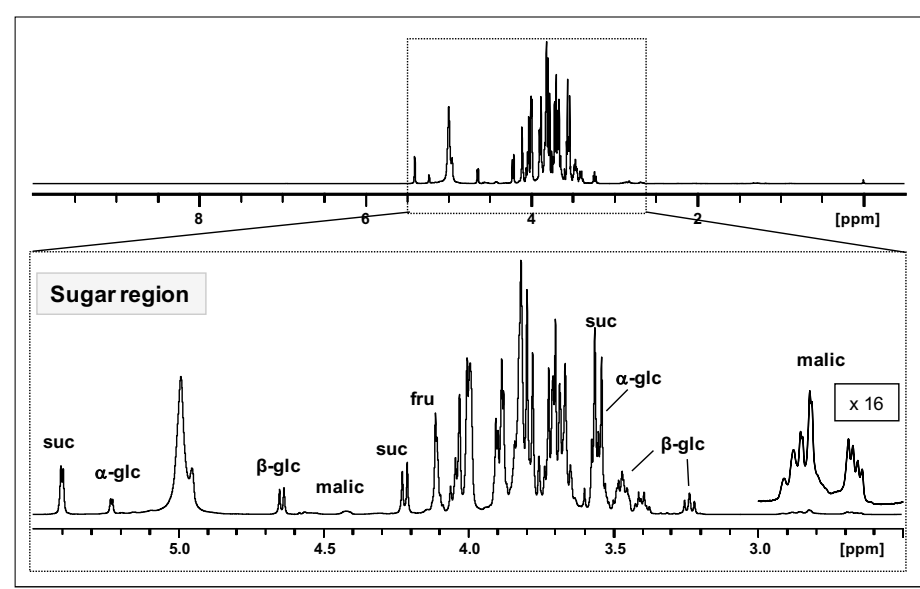

Fig. 2. ${ }^{1} \mathrm{H}$ HR-MAS NMR spectrum of apple tissue with an expansion of the sugar region (suc: sucrose, $\alpha$-glc: $\alpha$-glucose, $\beta$-glc: $\beta$-glucose, fru: fructose, malic: malic acid). 

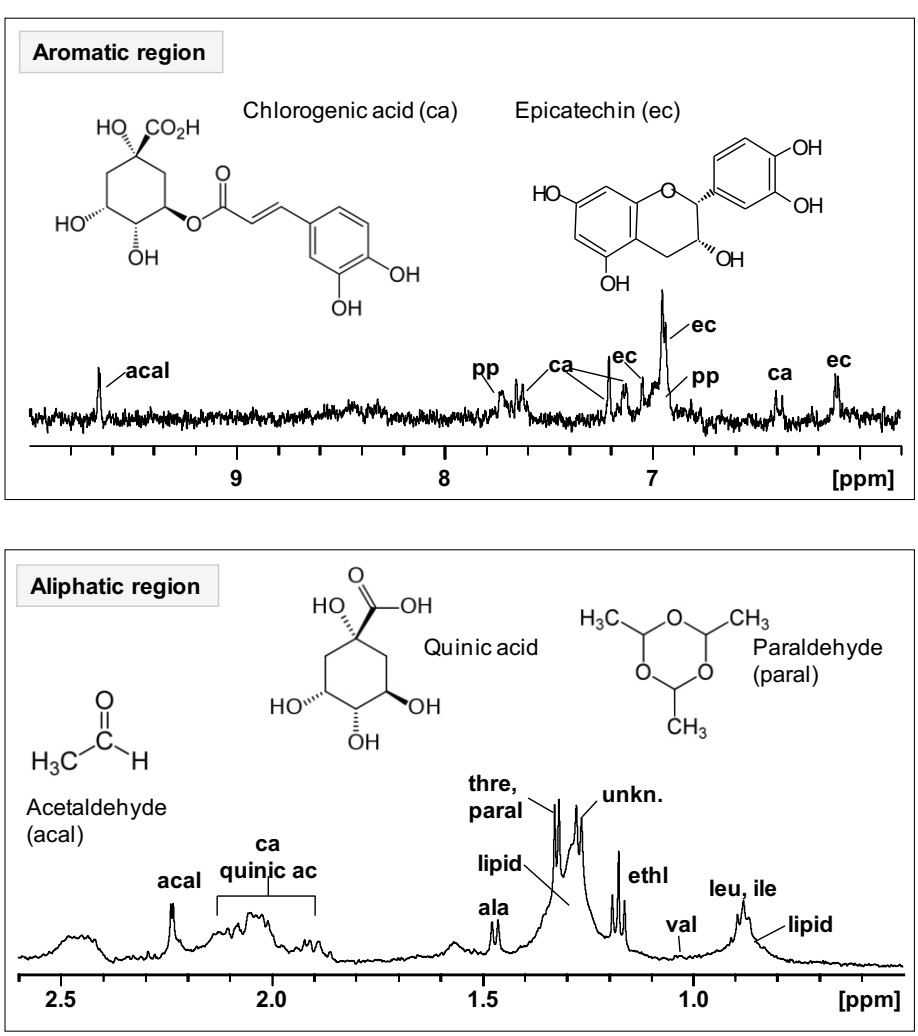

Fig. 3. ${ }^{1} \mathrm{H}$ HR-MAS NMR spectrum of apple tissue: expansion of the aromatic region (acal: acetaldehyde, pp: condensed polyphenols, ca: chlorogenic acid, ec: epicatechin).

Fig. 4. ${ }^{1} \mathrm{H}$ HR-MAS NMR spectrum of apple tissue with an expansion of the aliphatic region (acal: acetaldehyde, ca: chlorogenic acid, quinic ac: quinic acid, ala: alanine, thre: threoine, paral: paraldehyde, unkn: unknown, ethl: ethanol val: valine, leu: leucine, ile: isoleucine).

same apples and immediately measured. Several distinct differences could be observed between the pulp and juice spectra, which were most likely induced by the juice preparation process including tissue disruption and exposure to air. Besides an increase of the polyphenol signals in the juice, the resonances of ethanol, acetaldehyde and paraldehyde all present in the pulp disappeared in the juice while in turn formic and lactic acid occurred in the juice. In Fig. 5 details of a pulp and juice spectrum are shown along with the HR-MAS ${ }^{1} \mathrm{H}^{1} \mathrm{H}$-COSY spectrum of the pulp. While the amino acid signals of alanine, threonine and an unknown (1.27 ppm) were present in both, the cross peak of paraldehyde was missing in the juice. In conclusion, HR-MAS widely provides the metabolic profile of the genuine tissue composition while processes comprising tissue disruption induce changes specifically of those compounds sensitive towards oxidative reactions.

\section{Multivariate Statistical Analysis of HR-MAS-based NMR Data}

Besides a high reproducibility and quality of the raw data, multivariate statistical analysis of NMR spectra requires specific preparation steps to yield reliable interpretations. The most common steps usually applied in NMR-based metabonomics are summarized in Fig. 6. For our studies, a bucket width of $0.05 \mathrm{ppm}$ was applied while the regions where the malic acid signals appear were combined each into one larger bucket due to $\mathrm{pH}$-dependent shifts and the region of the residual water signal was excluded. The spectra were normalized to total integral accounting for differences in sample weights across the

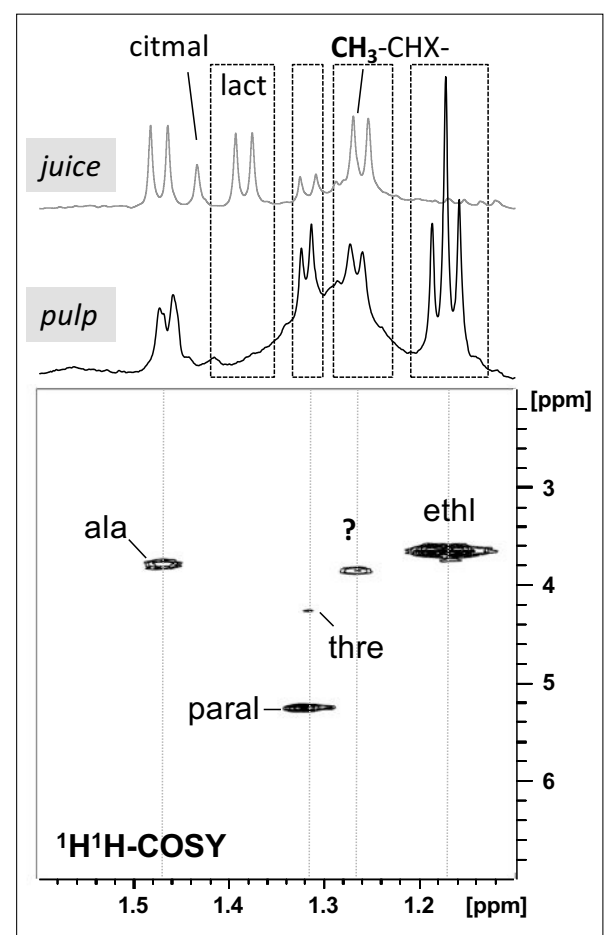

Fig. 5. Comparison of the ${ }^{1} \mathrm{H}$ HR-MAS NMR spectrum of apple tissue (bottom) with the liquid-state HR NMR spectrum of apple juice MAS COSY spectrum of apple tissue (citmal: citramalic acid, lact: lactic acid, ala: alanine, paral: paraldehyde, thre: threonine, ethl: ethanol). (top) obtained from the same apple. ${ }^{1} \mathrm{H}^{1} \mathrm{H} \mathrm{HR}-$ samples, and scaled to unit variance putting equal weight onto high and low intense components.

Apple metabolites are reported to be unevenly distributed across the fruit pulp due to differences in sun exposure during fruit development and compositional gradients from the core towards the peel.[26] Therefore, a standardized sampling was applied by punching 5 tissue probes from the same regions of each apple investigated. Moreover, in our first approach exploring the feasibility of HR-MAS NMR of apples for metabonomic studies, we aimed at assessing the variability of NMR data within single apples compared to the variability between apples. For this, 6 apples from 3 different cultivars, Golden delicious, Rubens and Braeburn, each were investigated comparing the $5 \mathrm{NMR}$ samples derived from the same apple with those derived from different apples within each cultivar group. The data were analyzed by means of principal component analysis (PCA) and analysis of variation (ANOVA) applied to defined spectral regions. The results indicated that the within apple variability was significantly lower than the between apple variability providing an important prerequisite for the subsequent chemometric studies. ${ }^{[24]}$

In a next step, the HR-MAS NMR data obtained from the apple tissue of the

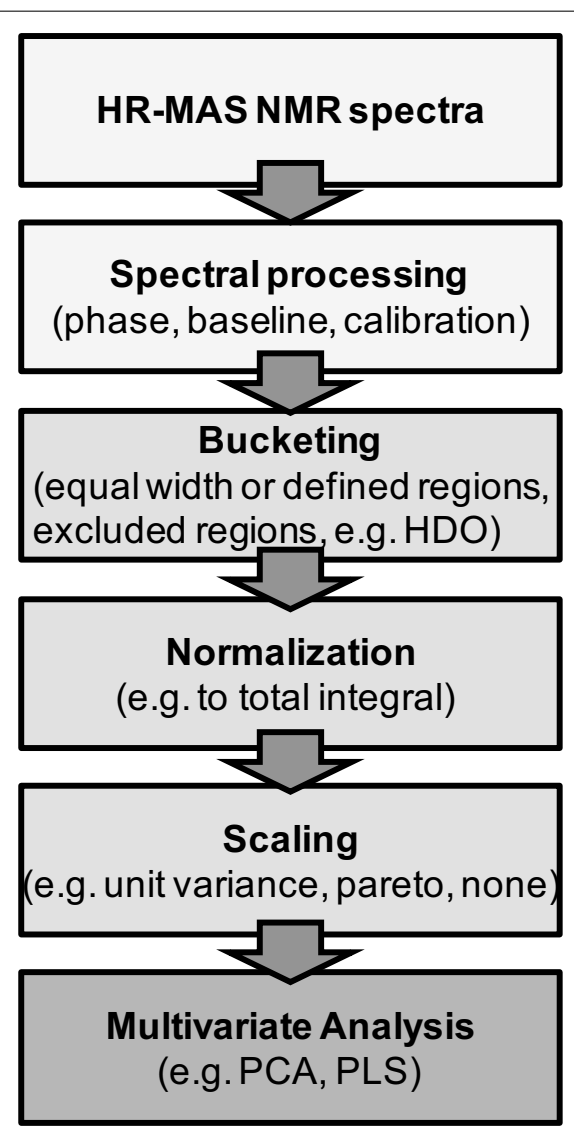

Fig. 6. Preparation steps involved in metabonomic analysis of NMR spectra. 


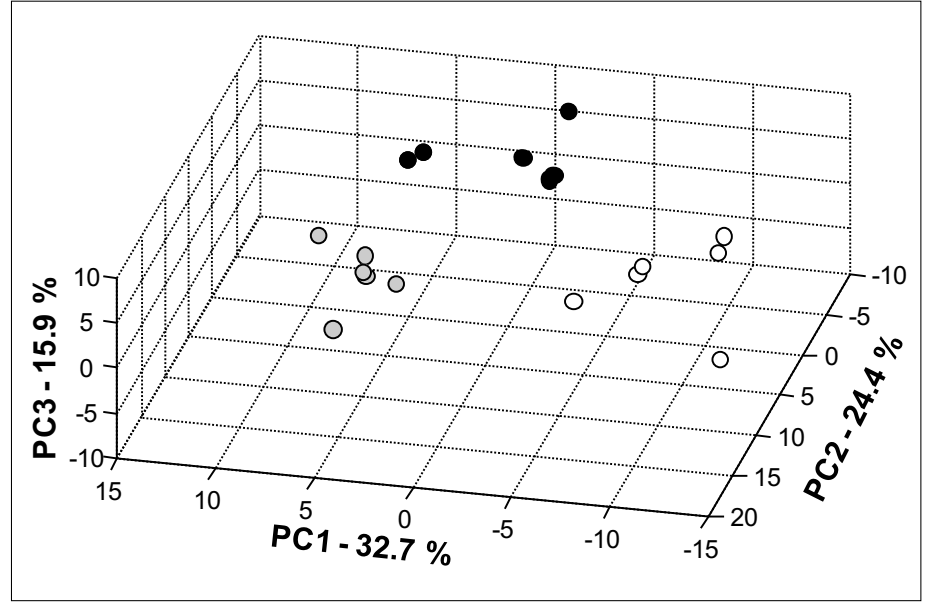

Fig. 7. 3D PCA scores plot PC1 vs PC2 vs PC3 for different apple cultivars. The PCA is based on 18 entries with each point representing the averaged spectrum obtained from 5 single spectra of one apple. Open circles: Golden Delicious, black: Rubens, grey: Braeburn.

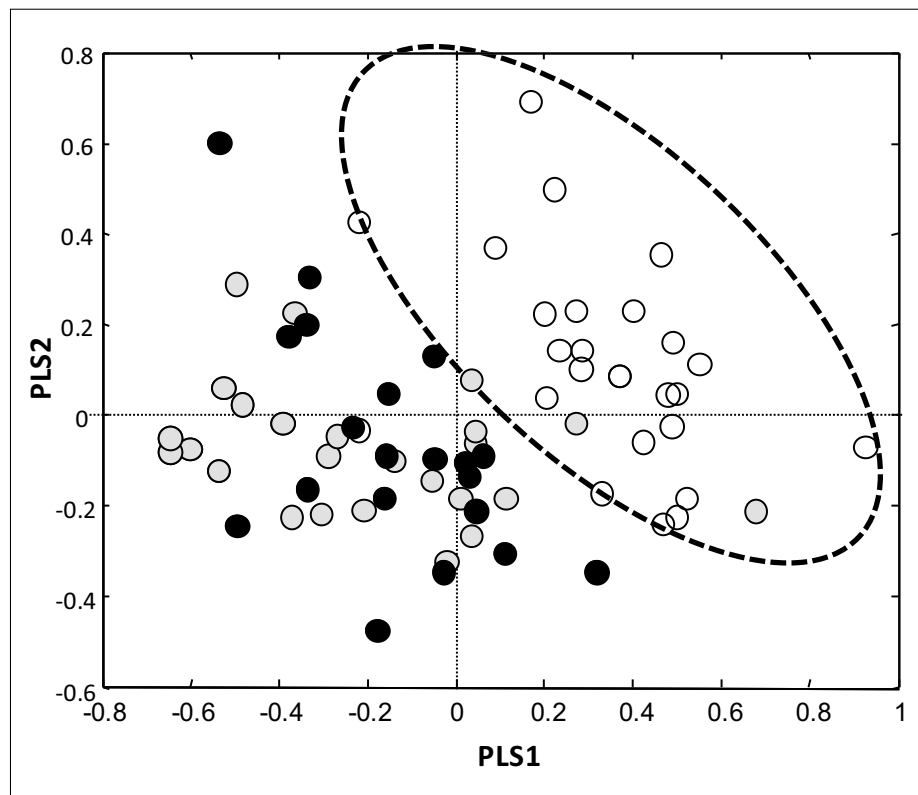

Fig. 8. Partial least squares discriminant analysis (PLS-DA) for apples derived from different cultivation methods. Open circles: Bio, black: integrated production (IP), grey: low-input production (LI). ${ }^{[27]}$

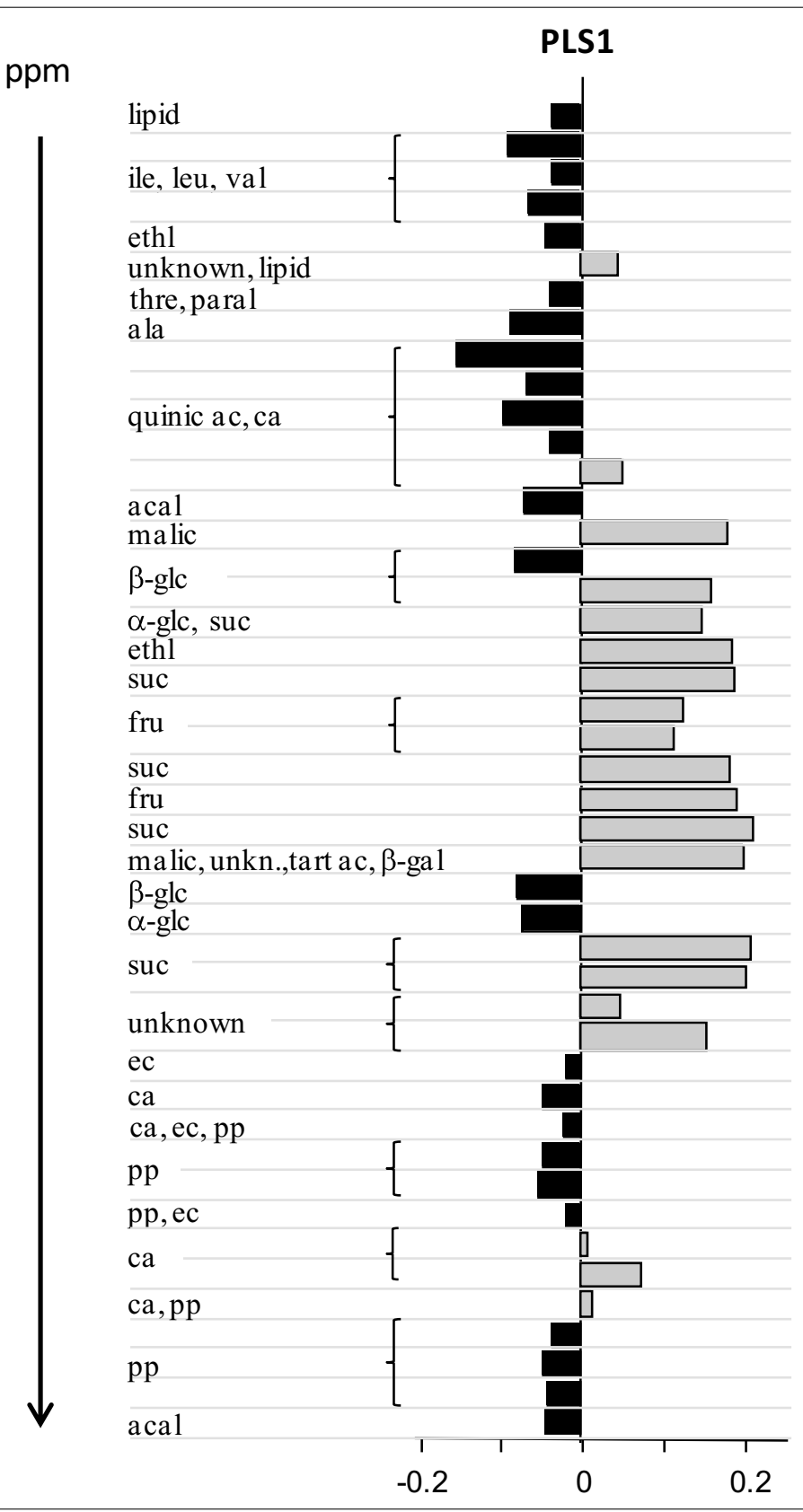

Fig. 9. Load values for the first PLS component and assigned buckets in the order of the ppm-scale. ${ }^{[27]}$
3 different cultivar groups, i.e. a total of 90 spectra, were analyzed by principal component analysis (PCA) and partial least squares discriminant analysis (PLSDA) to probe whether differences between groups could be observed. Both methods, PLS-DA based on spectral cultivar assignment, but also unsupervised PCA yielded a clear separation of the three cultivars. ${ }^{[24]}$ In Fig. 7, the 3D PCA scores plot for the first 3 principal components explaining $73 \%$ of the total variance is shown for the HR-MAS data of the three apple cultivars Golden Delicious, Rubens, and Braeburn. In this plot, each data point represents the average of 5 spectra derived from the same apple. Golden delicious and Braeburn mainly separated due to their differences along the first principal component (PC1) while they both discriminated from Rubens apples due to their different PC3 scores.

Based on our promising preliminary studies we are currently probing the impact of different cultivation methods on the metabolic profile of apples by HR-MAS NMR metabonomic studies. In collaboration with Agroscope Wädenswil (ACW), we have investigated Golden Delicious apples, which were grown applying three different plant protection strategies, i.e. organic growth conditions (Bio), the commonly applied integrated production (IP) and a low-input plant protection strategy (LI), which minimizes the pesticide use focusing on apple scab to which Golden Delicious apples are susceptible. Applying our previously developed standardized protocol, Bio apples could be well separated from the two remaining cultivation methods by PCA and PLS.[27] The corresponding PLS scores plot based on the analysis of 70 HR-MAS NMR spectra is shown for the first two PLS components in Fig. 8. Bio apples were mostly discriminated from LI and IP apples along PLS1. Analysis of the PLS load values provides information about which compounds mainly contribute to the discrimination. In Fig. 9, the load values of PLS1 are shown for those buckets which could be assigned to specific apple tissue components in the order of their spectral appearance (ppmscale). According to this plot, Bio apples are mainly discriminated due to their high 
levels of sucrose, fructose and malic acid, while LI and IP apples have higher levels of $\alpha$ - and $\beta$-glucose and quinic acid. There are also some contributions for the separation from the aromatic region with increased polyphenol levels in IP and LI apples.

The results of this study suggest that differences in the chemical composition are induced by the application of different cultivation methods. Thus, the proposed model may now serve as a starting basis for testing further apple samples obtained from subsequent harvest seasons for validation. Moreover, even though apples derived from the three different production systems all were harvested on the same day with a similar ripening index, it remains to be tested whether maturity differences contribute to the HR-MAS based discrimination. For this, we are currently investigating in an ongoing longitudinal HR-MAS NMR study apples from the three different cultivation methods as function of ripening index assessed by conventional analytical methods.

\section{Conclusions}

High-resolution magic-angle-spinning NMR spectroscopy provides a rapid and non-destructive analytical tool to obtain a comprehensive molecular fingerprint of the intact, unaffected apple tissue without the need for any preparation steps. The NMR data may thus help understand physiological processes in apples and how they are related to environmental factors like growth and storage conditions. This knowledge may in turn help to guide production processes of apples aimed at pro- viding year-round competitive high quality fruits. The HR-MAS studies applied to apples exemplify that the combination of HR-MAS NMR spectroscopy with multivariate statistical analysis has great potential as tool in the analysis of semi-solid food samples like fruits and vegetables, in general. This may become in particular of interest since factors like product identification, traceability and the detection of frauds play increasing roles in food quality control.

\section{Acknowledgement}

Financial support was obtained from the Swiss National Science Foundation (SNF) for the purchase of the HR-MAS probe, grant \# 206021-128736. The authors are grateful to Agroscope, Wädenswil (ACW), in particular to Dr. Franz Gasser, Dr. Daniel Baumgartner, and Dr. Claudia Good, for their inspiring and ongoing collaboration.

Received: August 10, 2012

[1] I. F. Duarte, A. M. Gil, Prog. Nucl. Mag. Res. Sp. 2012, 62, 51.

[2] J. S. McKenzie, J. A. Donarski, J. C. Wilson, A. J. Charlton, Prog. Nucl. Mag. Res. Sp. 2011, 59 , 336.

[3] W. Kolodziejski, J. Klinowski, Chem. Rev. 2002, 102, 613.

[4] M. J. Shapiro, J. S. Gounarides, Biotechnol. Bioeng. 2000, 71, 130.

[5] L. H. Tseng, D. Emeis, M. Raitza, H. Handel, K. Albert, Z. Naturforsch. B-A J. Chem. Sci. 2000, $55,651$.

[6] A. Pampel, F. Volke, Lecture Notes in Physics 2004, 634, 439.

[7] M. Santoro, P. Marchetti, F. Rossi, G. Perale, F Castiglione, A. Mele, M. Masi, J. Phys. Chem. $B$ 2011, 115, 2503

[8] M. Valentini, M. Ritota, C. Cafiero, S. Cozzolino, L. Leita, P. Sequi, Magn. Reson. Chem. 2011, 49, S121.

[9] J. C. Lindon, O. P. Beckonert, E. Holmes, J. K. Nicholson, Prog. Nucl. Mag. Res. Sp. 2009, 55, 79.
[10] M. Coen, P. W. Kuchel, Chem. Aust. 2004, 71, 13.

[11] J. K. Nicholson, J. C. Lindon, Nature 2008, 455, 1054.

[12] T. Ebbels, J. Lindon, M. Coen, 'Processing and Modeling of Nuclear Magnetic Resonance (NMR) Metabolic Profiles', in 'Metabolic Profiling', 708 edn., Ed. T. O. Metz, Humana Press, 2011, pp 365-388.

[13] G. del Campo, J. I. Santos, N. Iturriza, I Berregi, A. Munduate, J. Agric. Food Chem. 2006, 54, 3095.

[14] M. Spraul, B. Schutz, E. Humpfer, M. Mortter, H. Schafer, S. Koswig, P. Rinke, Magn. Reson. Chem. 2009, 47, S130.

[15] C. Almeida, I. F. Duarte, A. Barros, J Rodrigues, M. Spraul, A. M. Gil, J. Agric. Food Chem. 2006, 54, 700.

[16] G. Imparato, E. Di Paolo, A. Braca, R. Lamanna, J. Agric. Food Chem. 2011, 59, 4429.

[17] L. Shintu, S. Caldarelli, J. Agric. Food Chem. 2006, $54,4148$.

[18] D. Sacco, M. A. Brescia, A. Buccolieri, A. C. Jambrenghi, Meat Sci. 2005, 71, 542.

[19] C. M. Kingston, 'Maturity Indices for Apple and Pear', in 'Horticultural Reviews', John Wiley \& Sons, Inc., 1992, pp 407-432.

[20] T. Fuleki, E. Pelayo, R. B. Palabay, J. Agric. Food Chem. 1995, 43, 598.

[21] U. Vrhovsek, A. Rigo, D. Tonon, F. Mattivi, J. Agric. Food Chem. 2004, 52, 6532.

[22] S. Bureau, I. Scibisz, C. Le Bourvellec, C. M. G. C. Renard, J. Agric. Food Chem. 2012, 60, 3551.

[23] D. F. Holderbaum, T. Kon, T. Kudo, M. P. Guerra, HortScience 2010, 45, 1150.

[24] M. Vermathen, M. Marzorati, D. Baumgartner, C. Good, P. Vermathen, J. Agric. Food. Chem. 2011, 59, 12784.

[25] H.-D. Belitz, W. Grosch, P. Schieberle, 'Food Chemistry', 4th edn., Springer, 2009.

[26] Y. Z. Zhang, P. M. Li, L. L. Cheng, Food Chem. 2010, 123, 1013.

[27] M. Vermathen, M. Marzorati, D. Baumgartner, C. Good, P. Vermathen, 'Magnetic Resonance in Food Science: Food for Thought', Proceedings of the 11th Intl. Conference on Applications of Magn. Res. in Food Science, Royal Soc. Chemistry, Thomas Graham House, Science Park, Cambridge CB4 4WF, Cambs, England, 2012, in press. 